\title{
Esthetic Management of Dental Fluorosis: A Minimally Invasive Approach
}

\author{
Asma Nakhli' ${ }^{1,2}$, Farah Chaouali, ${ }^{1,2}$, Marwa Amara ${ }^{1,2}$, Sarra Nasri, ${ }^{1,2 *}$, \\ Zohra Nouira $^{1,2}$, Belhassan Harzallah ${ }^{1,2}$, Mounir Cherif ${ }^{1,2}$ and Dalenda \\ Hadyaoui $^{1,2}$ \\ ${ }^{1}$ Department of Fixed Prosthodontics in the Dentistry Clinic of Monastir, University \\ of Monastir, Tunisia \\ ${ }^{2}$ Research Laboratory of Occlusodontics and Ceramic Prostheses LR16ES15, \\ Monastir, Tunisia \\ *Corresponding Author: Sarra Nasri, Department of Fixed Prosthodontics in the \\ Dentistry Clinic of \\ Monastir, University of Monastir, Research Laboratory of Occlusodontics and \\ Ceramic Prostheses \\ LR16ES15, Monastir, Tunisia.
}

\begin{abstract}
Fluoride is known to be beneficial to teeth. However, when they are exposed to excessively, especially in children during odontogenesis, it can lead to fluorosis.

Fluorosis is a condition defined by a hypo-mineralization of the enamel characterized by coloration, which can turn into cavities and leads to an unsightly aesthetic appearance.

In Tunisia, fluorosis is a real scourge that affects certain regions where drinking-water contains excessive fluorine. The management of these patients differs depending on the extent of the damage. In this article, we will describe the minimally invasive management of a case with severe fluorosis.
\end{abstract}

Keywords: Dental Fluorosis; Micro Abrasion; Ceramic Veneers; Mockup; Bonding

\section{Introduction}

Dental fluorosis is an irreversible condition defined as hypomineralisation of enamel resulting from excessive ingestion of fluoride during tooth development [1-3]. It is characterized by discoloration, which can turn into cavitation leading to an unsightly aesthetic appearance and a real handicap for patients [4].

In several countries, dental fluorosis is defined as a public health problem [5]. In Tunisia, it presents a real scourge that affects certain regions where drinking water contains excessive fluoride [6].

The esthetic management of patients with fluorosis depends on the extent of the damage.
Received: December 27, 2021

Published: January 31, 2022

(C) All rights are reserved by Sarra Nasri., et al.
According to Dean's index, dental fluorosis can be classified into three main types: mild, moderate and severe.

Mild dental fluorosis is barely noticeable with only white striations. Moderate and severe dental fluorosis present contrariwise a mottled enamel with brownish discolorations that can proceed into pits and wear on the enamel surface due to pour enamel mineralization.

Different treatment options have been proposed such as bleaching, resin infiltration, micro abrasion, macro-abrasion, composite restorations, ceramic veneers and conventional prosthetic procedures [7-9]. 
In this article, we will describe the case of a patient from Kairouan who presented with a severe fluorosis and was successfully treated with a minimally invasive esthetic technique including micro abrasion then ceramic veneers.

\section{Case Report}

A 23-year-old Tunisian female was referred to the department of fixed Prosthodontics in the dental clinic of Monastir. Her chief complaint was an unpleasant smile caused by generalized tooth discoloration. She lived in the region of Kairouan that is located in the center of Tunisia where high levels of natural fluoride can be found in drinking water.

Clinical examination revealed a high smile line uncovering less than $2 \mathrm{~mm}$ of gum tissue.

Her smile is wide, exposing teeth between the maxillary second premolars. She had not previously undergone treatment to address this complaint (Figure 1).

Oral examination revealed generalized fluorosis. Based on the Dean Index for dental fluorosis classification, this case was classified as class 4 .

White and brown spots were described on all teeth. They were more pronounced on the maxillary central incisors with moderate loss of tooth substance. The teeth 11 and 21 presented restorations with composite resin.

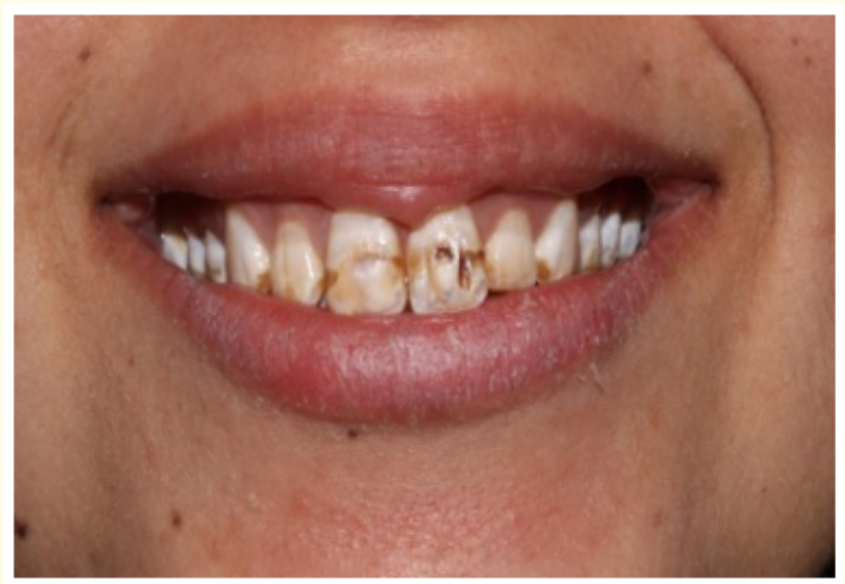

Figure 1: Pre treatment situation.
The patient demonstrated a good oral hygiene. Occlusal examination revealed bilateral Class 1 with vertical and horizontal overlaps of $3 \mathrm{~mm}$. The radiographic examination showed no caries or bone loss.

After the clinical examination, all available treatment options were presented to the patient. Since she desired a long-term solution, she opted for ceramic veneers for her upper teeth. Given its limited financial capacity, she decided to postpone the treatment of her lower teeth.

In order to enhance esthetic result under ceramic veneers, sessions of micro-abrasion were decided (Figure 2).

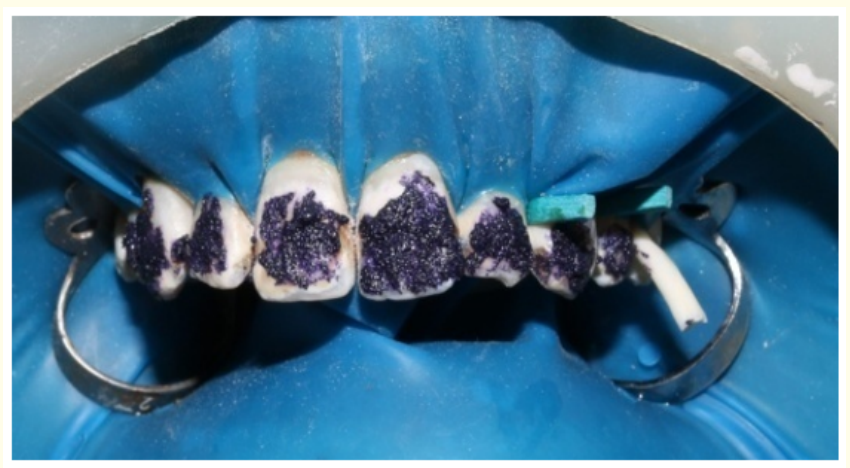

Figure 2: Microabrasion.

After rubber dam application, micro-abrasion was performed with an abrasive paste containing silicon carbide particles and $6.6 \%$ hypochloric acid slurry (Opalustre ${ }^{\circledR}$, Ultradent Products, Inc South Jordan UT, USA).

A layer is applied only on the discolored area using specific rubber cup (Opalcup Ultradent Products, Inc South Jordan UT, USA), attached to a contra angle handpiece. A chair session was performed with 10 cycles of 10 seconds each and $20 \mathrm{sec}$ of rinsing between each application.

After each cycle an evaluation was done to decide whether to proceed to another application. Finally, fluoride varnish was applied (8) (Figure 3).

Study cast and photographs were realized and a detailed smile analysis was performed using a smile design tool (Powerpoint ${ }^{\circledR}$ templates). The virtual planning was presented to the patient, but 


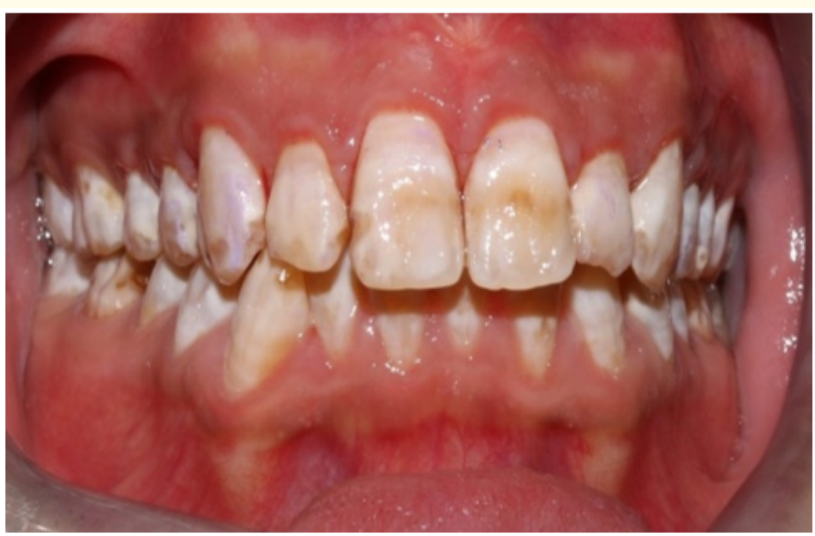

Figure 3: Result after microabrasion.

it was insufficient to judge the future restorations. Interim prostheses (mock-up) are of paramount importance to visualize the definitive restorations and provide better communication. A wax-up on the study model was performed to correct the shape and position of the teeth (Figure 4).

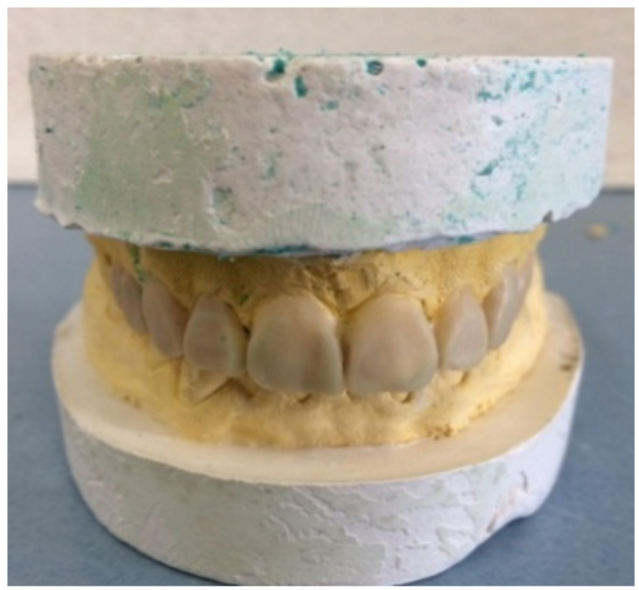

Figure 4: Waxed diagnostic cast.

A polydimethylsiloxane material (Mono Ghenesyl, Lascod, Firenze, Italy) impression of the diagnostic wax-up was made to fabricate a silicone index. Bis-acrylic resin (Dentocrown automix Itena, Villepint, France) was then inserted into the silicone index to transfer the diagnostic wax-up to interim prostheses in the patient mouth (Figure 5).
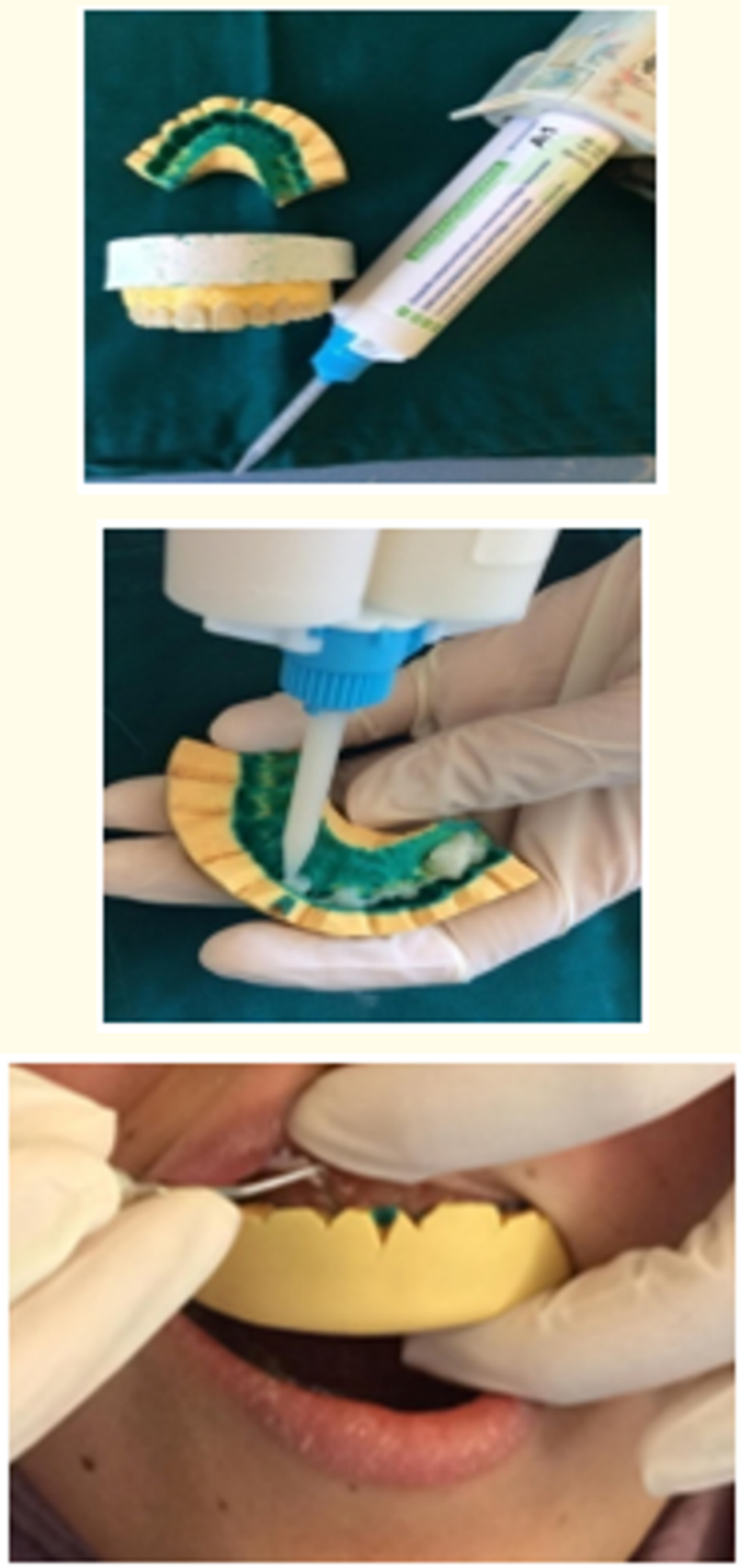

Figure 5: Mock-up.

The interim prostheses allowed clinicians and patient to fully evaluate the suggested anatomy and esthetics for the definitive restorations (Figure 6) and were adjusted for optimized occlusion, phonetics, and esthetic 


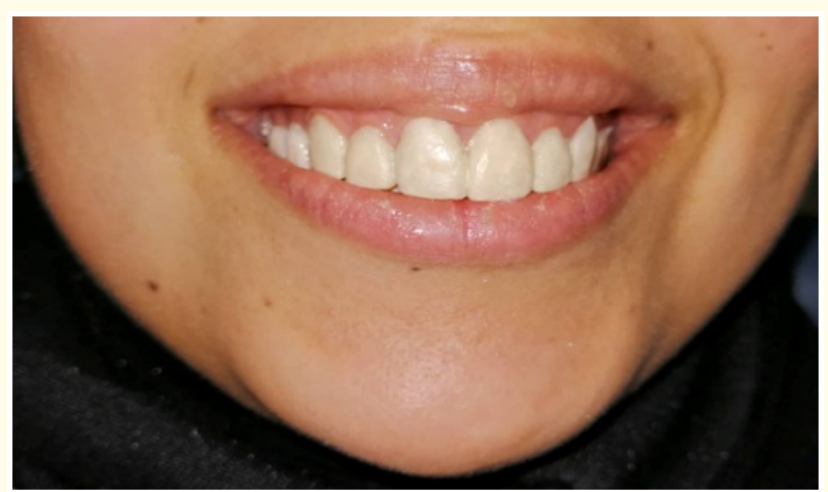

Figure 6: Intraoral views with the mock-up.

All needed modifications were performed at this time. After patient approval, all modifications were transferred to the wax-up to assist the fabrication of the porcelain laminate veneers.

A minimally invasive approach was performed for tooth preparation. Depth grooves using depth burs were made on the labial surface of the mock-up. As for incisal edge, depth grooves were marked using a round burs. Predetermined depths were prepared with diamond rotary instruments (FG 3053 and FG 2134; KG Sorensen, Cotia, Brazil) according to the thickness required for each laminate veneer (Figure 7). Tooth preparation with the interim prostheses in place allowed a small amount of enamel tissue removal ( 0.3 to $0.5 \mathrm{~mm}$ for each tooth).
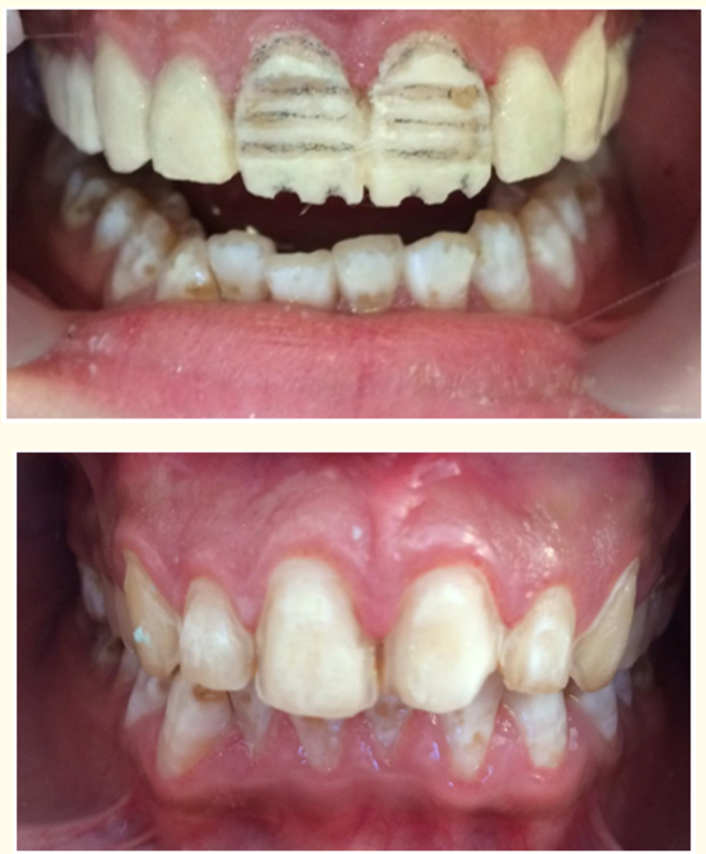

Figure 7: Tooth preparation.
After complete removal of the interim prostheses, final preparation depth was concluded, and finishing procedures were performed using carbide finishing burs (FG 151Z; KG Sorensen).

A single retraction cord (Ultrapak Cord \#0; Ultradent Products, Inc., South Jordan, UT) was used for mechanical gingival displacement before digital impression with Crestream $₫ 3600$ intraoral scanner (Figure 8).

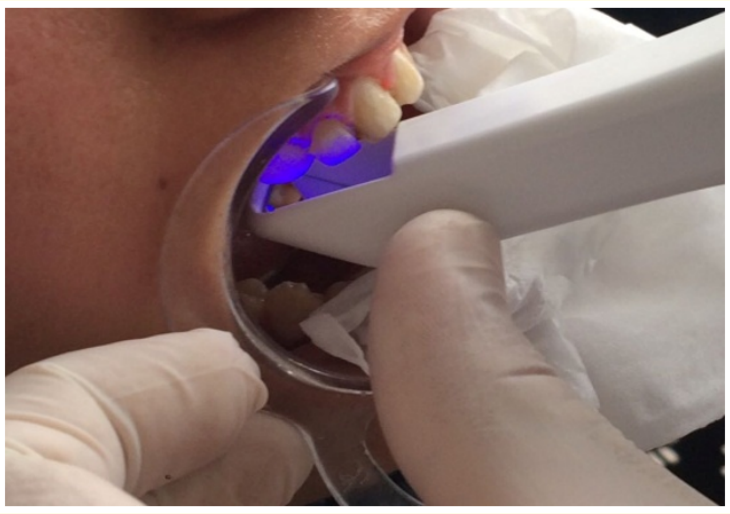

Figure 8: Digital impression.

Laminate veneers of lithium disilicate (IPS e.max; Ivoclar Vivadent, Schaan, Liechtenstein) were fabricated in the chosen tooth color (A1) (Figure 9).

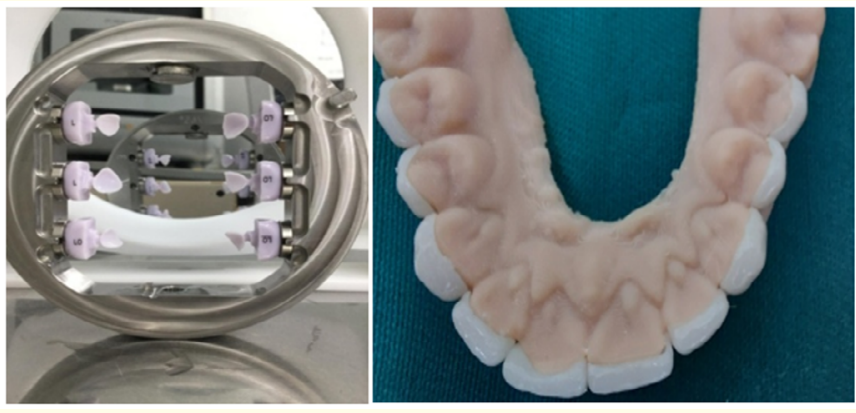

Figure 9: Veneers fabrication.

Function, form, and phonetics of the restorations were satisfactory, and the patient was satisfied with the final esthetic outcome (Figure 10).

\section{Discussion}

Fluorosis is endemic in certain regions of Tunisia, particularly Kairouan, with a prevalence of $57.7 \%$ among children at the age 


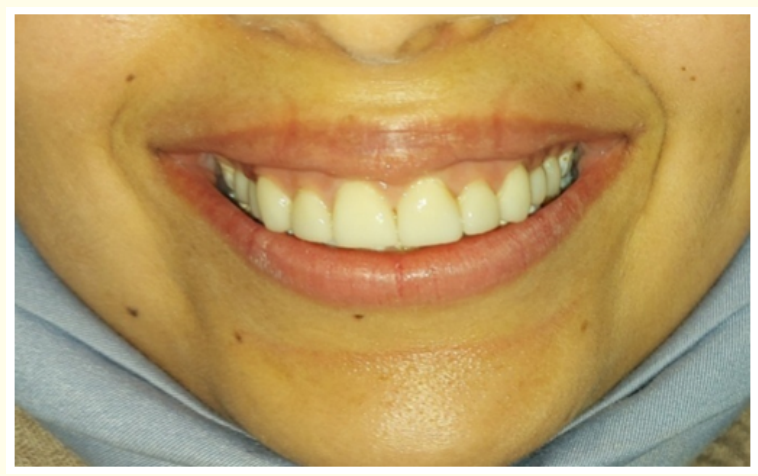

Figure 10: Post Treatment view.

of 12 . The rate of fluorosis increases with the increase of fluoride content in drinking water.

The esthetic management of patients with fluorosis depends on the extent of the damage.

Bleaching alone or in combination with micro/macro abrasion provides a satisfactory improvement in the aesthetic appearance of teeth with mild or moderate fluorosis $[7,8]$.

Composite veneers provide good esthetic outcome and patient's satisfaction; however, because of its physical properties and to the bonding strength when compared, they cannot be considered as an ultimate solution for severe as well as some cases of moderate fluorosis. In these conditions, either veneers or full coverage crowns are needed to satisfy the patient's esthetic demands. Enamel preservation and careful treatment planning are considered two of the most important factors to achieve clinical success in laminate veneers $[10,11]$. Proper material selection for esthetic veneers is one of the most important decisions to be made by clinicians. It affects the long-term function, longevity, and esthetics of restorations. There are a large number of restorative materials available, which has increased the complexity of the decision-making process [12]. For anterior restorations like in our case, a translucent ceramic material with lower mechanical strength lead to favorable esthetic outcomes. Lithium disilicate glass ceramic was chosen for its biocompatibility, high mechanical properties in addition to its very good esthetic features, especially as regards translucency, that is about $30 \%$ higher than conventional zirconia [13].

Digital impression using intraoral scanners have multiple advantages compared to conventional methods. They are less time consuming, more ergonomic and more comfortable for the patients.

However, while the accuracy of intraoral scanner systems appears to be promising and comparable to conventional methods, they are still vulnerable to inaccuracies [14].

As for bonding protocol, in view of the compromised bonding surfaces of fluorosed enamel some authors recommend a double etching protocol in order to improve bond strength. Otherwise, according to comparative in vitro studies of the simple and the double etching protocol, there were no significant differences observed between the two protocols in moderately and severely fluorosed teeth $[15,16]$.

\section{Conclusion}

- Dental fluorosis is a common disorder that can manifest in a variety of clinical presentations. The esthetic consequences of dental fluorosis can negatively affect quality of life.

- In severe cases, where the fluorosed enamel is prone to chipping, minimally invasive procedures are ineffectual and a more substantial restorative approach is required to restore optimal function and esthetics. Thus, the appropriate treatment decision must be reached through a thorough evaluation of the conditions of the affected teeth, as well as the overall functional and esthetic needs of the patient.

- However, no definitive guidelines exist for the management and treatment of severe dental fluorosis due to the limited evidence available in the literature.

\section{Bibliography}

1. Ayoob S and Gupta AK. "Fluoride in Drinking Water: A Review on the Status and Stress Effects". Critical Reviews in Environmental Science and Technology 36.6 (2006): 433-487.

2. Farias-Neto A., et al. "Tooth preparation for ceramic veneers: when less is more". International Journal of Esthetic Dentistry 14.2 (2019): 156-164.

3. Farías P., et al. "Fluoride Exposure through Different Drinking Water Sources in a Contaminated Basin in Guanajuato, Mexico: A Deterministic Human Health Risk Assessment". International Journal of Environmental Research and Public Health 18.21 (2021): 11490. 
4. Silva MCC., et al. "Effect of fluoridated water on dental caries and fluorosis in schoolchildren who use fluoridated dentifrice". Brazilian Dental Journal 32.3 (2021): 75-83.

5. Saldarriaga A., et al. "Clinical changes in the severity of dental fluorosis: a longitudinal evaluation". BMC Oral Health 21.1 (2021): 366.

6. Maatouk F. "School oral health survey in Kairouan, Tunisia" (1998).

7. Wang Q., et al. "Minimally invasive esthetic management of dental fluorosis: a case report". The Journal of International Medical Research 48.10 (2020): 300060520967538.

8. Romero MF., et al. "Minimally invasive esthetic improvement in a patient with dental fluorosis by using microabrasion and bleaching: A clinical report". Journal of Prosthetic Dentistry 120.3 (2018): 323-326.

9. Ardu S., et al. "A minimally invasive treatment of severe dental fluorosis". Quintessence International (Berlin, Germany: 1985) 38.6 (2007): 455-458.

10. Gurel G., et al. "Influence of enamel preservation on failure rates of porcelain laminate veneers". The International Journal of Periodontics and Restorative Dentistry 33.1 (2013): 31-39.

11. Oztürk E and Bolay S. "Survival of porcelain laminate veneers with different degrees of dentin exposure: 2-year clinical results". The Journal of Adhesive Dentistry 16.5 (2014): 481-489.

12. Moshaverinia A. "Review of the Modern Dental Ceramic Restorative Materials for Esthetic Dentistry in the Minimally Invasive Age". Dental Clinics of North America 64.4 (2020): 621631.

13. Baldissara P., et al. "Translucency of zirconia copings made with different CAD/CAM systems". Journal of Prosthetic Dentistry 104.1 (2010): 6-12.

14. Aboushelib MN., et al. "Microtensile bond strength of different components of core veneered all-ceramic restorations. Part 3: double veneer technique". Journal of Prosthodontics: Official Journal of the American College of Prosthodontists 17.1 (2008): 9-13.

15. Isci D., et al. "Effects of fluorosis on the shear bond strength of orthodontic brackets bonded with a self-etching primer". European Journal of Orthodontics 33.2 (2010): 161-166.
16. Akpata E. "Therapeutic management of dental fluorosis: A critical review of literature". Saudi Journal of Oral Sciences 1.1 (2014): 3-13.

\section{Assets from publication with us}

- Prompt Acknowledgement after receiving the article

- Thorough Double blinded peer review

- Rapid Publication

- Issue of Publication Certificate

- High visibility of your Published work

Website: www.actascientific.com/

Submit Article: www.actascientific.com/submission.php Email us: editor@actascientific.com

Contact us: +919182824667 